\title{
Orthognathic treatment of facial asymmetry due to temporomandibular joint ankylosis
}

\author{
Ayse Gulsen ${ }^{1}$, Serhat Sibar ${ }^{2}$, Selahattin Ozmen ${ }^{3}$ \\ ${ }^{1}$ Department of Plastic, Reconstructive, and Aesthetic Surgery, Gazi University Hospital, Ankara; ${ }^{2}$ Department of Plastic, Reconstructive, and \\ Aesthetic Surgery, Duzce Ataturk State Hospital, Duzce; ${ }^{3}$ Department of Plastic, Reconstructive, and Aesthetic Surgery, Koc University \\ Hospital, Istanbul, Turkey
}

The aim of this study was to present a case series of the orthognathic treatment of facial asymmetry due to temporomandibular joint (TMJ) ankylosis and to characterize the current treatment modalities through a literature review. Four patients who presented with facial asymmetry due to TMJ ankylosis between 2010 and 2014 were included in this study. TMJ ankylosis was surgically treated before bimaxillary surgery with advancement genioplasty in some of the cases. In 2 cases, 3-dimensional (3D) models were used for diagnosis and treatment planning, as 3D models are very important tools for planning surgical maneuvers. Aesthetically pleasant facial symmetry and a good facial profile were obtained in all the cases.

Keywords Cephalometry / Facial asymmetry / Maxillofacial development / Orthognathic surgery / Temporomandibular joint
Correspondence: Serhat Sibar Department of Plastic, Reconstructive, and Aesthetic Surgery, Duzce Ataturk State Hospital, Aziziye Mh., Merkez, 81010 Duzce, Turkey

Tel: +90-380-529-1300

Fax: $+90-380-529-1328$

E-mail: serhatsibar@hotmail.com

Received: 2 Oct 2016 • Revised: 5 Feb 2017 • Accepted: 21 Mar 2017

pISSN: 2234-6163 • elSSN: 2234-6171 • https://doi.org/10.5999/aps.2016.01774• Arch Plast Surg 2018;45:74-79

\section{INTRODUCTION}

Facial asymmetry is a 3-dimensional (3D) problem first observed by Greek artists that can have a negative impact on facial harmony, attractiveness, and beauty [1]. According to some authors, natural facial asymmetry (defined as "normal") becomes "abnormal" when the lateral deviation is greater than $2 \mathrm{~mm}$, which is described as the critical value [2].

Thus far, facial asymmetry has been attributed to genetic factors (e.g., multiple neurofibromatosis and hemifacial microsomia) and environmental factors (e.g., trauma, functional deviations, and mandibular shifts a result of tooth interference) [3]. Unilateral temporomandibular joint (TMJ) ankylosis causes facial asymmetry due to the undergrowth of the mandible.

TMJ ankylosis is a fusion in the TMJ area that restricts jaw movements. This restriction varies from slight interference to a complete inability to open the jaw [4].
In this case report, the results of orthognathic surgery performed in 4 patients with severe facial asymmetry due to TMJ ankylosis are presented.

In cases 1 and 4, 3D models were used for the diagnosis and surgical treatment planning.

All patients were informed before the study about the aims of the procedure and associated risks, and their consent was obtained prior to participating in the study.

\section{CASES}

\section{Case 1}

A 16-year-old female patient was admitted to our department. She had a history of TMJ ankylosis with limitations in opening her mouth on the left side due to the trauma that she had experienced when she was 5 years old. After a diagnosis with plain Xray and computed tomography (CT), the patient underwent 
surgery to release the ankylosis. She stated that her preoperative $10-\mathrm{mm}$ mouth opening became $20 \mathrm{~mm}$ after the surgery. The patient then used a functional appliance (a hybrid appliance) to maintain the mouth opening range and to correct her facial asymmetry. At the age of 11 years, her facial asymmetry became persistent, with an increasingly retrognathic lower chin appearance during the growth period. Fixed orthodontic appliances were then used for correcting the alignment problem. When she presented at our clinic, the alignment of her teeth had already been completed. A clinical examination indicated the presence of mandibular deviation to the left side, maxillomandibular deviation to the left side, occlusal tilting, and a bird-like facial appearance due to the retrognathic mandible (Fig. 1A). In the intraoral examination, an increased overjet and class II malocclusion were noted. In the cephalometric evaluation, bimaxillary retrusion (sella-nasion-A point [SNA]: $77^{\circ}$, sella-nasion-B point [SNB]: $66.5^{\circ}$ ) with a class II skeletal relationship (A point-nasion-B point $\left.[\mathrm{ANB}]: 11.5^{\circ}\right)$ and high mandibular plane inclination (sella-nasion/gonion-gnathion [SN/GoGn]: $40^{\circ}$ ), midline deviation, and asymmetric ramal heights were observed (Table 1). A 3D model was created using the patient's CT images (Fig. 2). An evaluation of the $3 \mathrm{D}$ images revealed that the mandibular ramal heights (condyle-gonion [Co-Go]) were $55 \mathrm{~mm}$ on the right side and $45.5 \mathrm{~mm}$ on the left. The mandibular body length was $73 \mathrm{~mm}$ on the right and $56.5 \mathrm{~mm}$ on the left. The maxillary heights between the left and the right orbital point and the maxillary first molars were $46.5 \mathrm{~mm}$ and $38.5 \mathrm{~mm}$, respectively. Characteristic antegonial notching on the left was easily detectable on the 3D model (Fig. 2).

\section{Surgical plan}

(1) Correction of maxillary tilting with impaction of the right segment $(4 \mathrm{~mm})$ and extrusion of the left segment $(4 \mathrm{~mm})$ at

\section{Fig. 1. Extraoral photographs of case 1}

Before (A) and after (B) surgery.
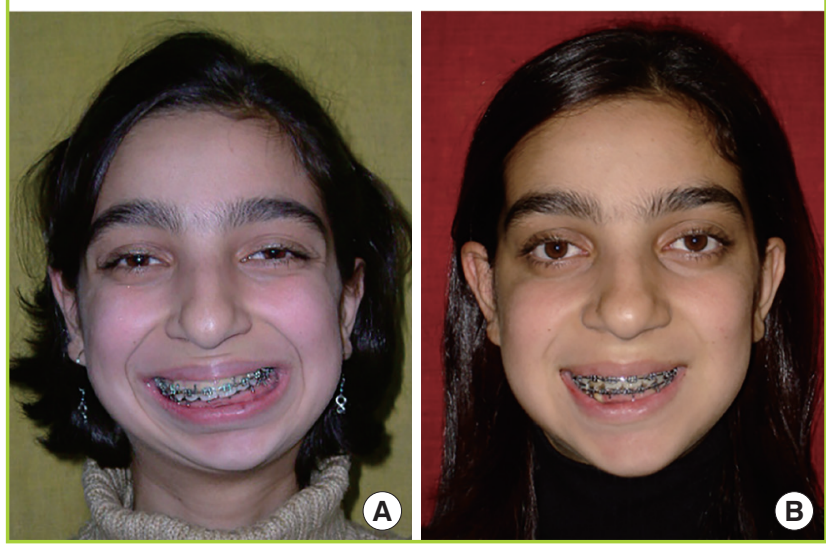

the molar tooth level; 2-mm maxillary advancement in the anteroposterior direction.

(2) Advancement of the mandible and chin with bilateral sagittal split osteotomy (BSSO) and advancement genioplasty.

The treatment plan was first simulated on the 3D model of the patient. Following surgery, a fixed appliance was maintained and 4-week intermaxillary fixation was performed. During the1-year follow-up, no relapse was observed. After the surgery, the patient demonstrated a more symmetrical facial appearance, a balanced smile-teeth relationship, and a better lateral profile (Fig. 1B).

\section{Case 2}

The second patient was 17-year-old when she presented at our department. Her medical history revealed TMJ ankylosis on her right due to a ear infection and the development of facial asymmetry. Her maximum mouth opening was reported to be 12 $\mathrm{mm}$ after the TMJ ankylosis surgery. She then used a functional orthodontic appliance. After a year, her mouth opening was reported to be $18.5 \mathrm{~mm}$ and the correction of the midline deviation was $5 \mathrm{~mm}$. Clinically, facial asymmetry with a deviation to the right, a bird-like facial appearance, difficulty in lip closure, tilted occlusion, inequality of gingival show during smiling, a retrognathic mandibular appearance, a large overjet, and Angle class II dental malocclusion were observed (Fig. 3A). In the cephalometric analysis using a lateral cephalogram, bimaxillary retrognathia (SNA: $\left.75^{\circ}, \mathrm{SNB}: 68^{\circ}\right)$ with a skeletal class II maxillomandibular relationship (ANB: $7^{\circ}$ ), and proclination of the upper and lower incisal teeth, were observed (Table 1). In the posteroanterior cephalogram, the discrepancy between the left and the right maxillary height, and between the molar teeth and the orbital point was $6.5 \mathrm{~mm}$. The discrepancy between the left

\section{Fig. 2. Three-dimensional model of case 1}

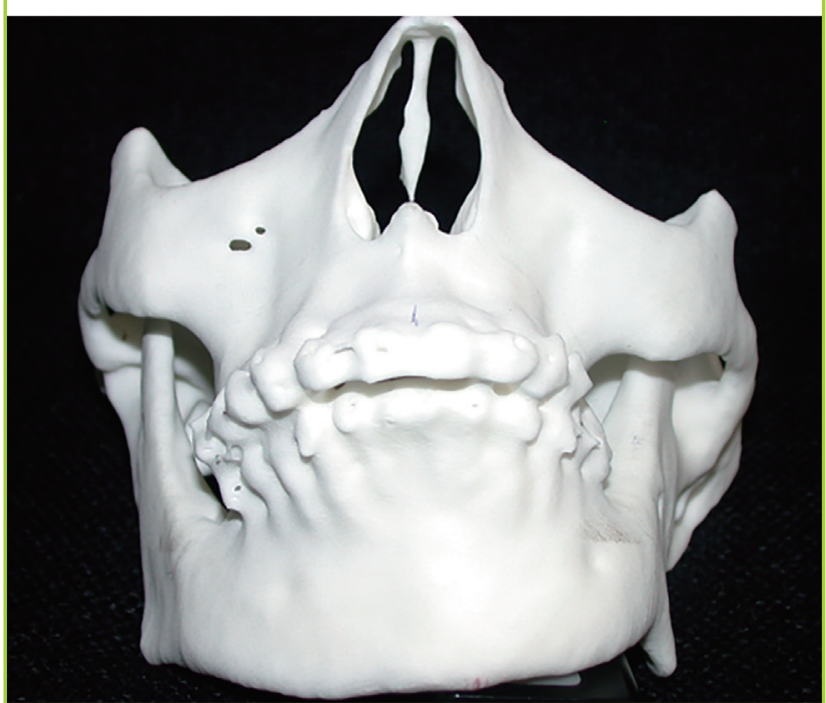



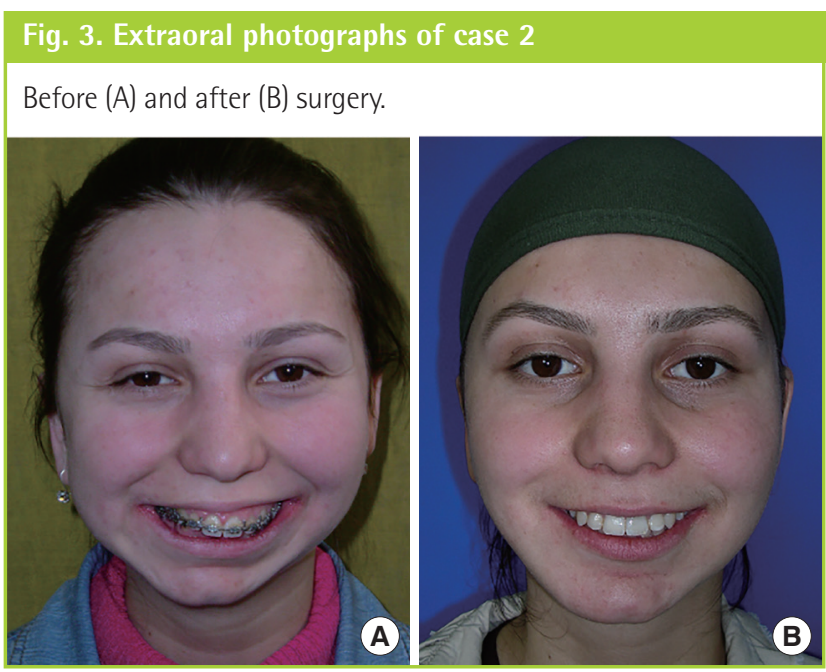

and right mandibular ramal length was $8.5 \mathrm{~mm}$, and the deviation in the menton from the midline was $10 \mathrm{~mm}$ (Table 1). Deep antegonial notching was noted in the cephalograms.

\section{Surgical plan}

Le Fort 1 osteotomy to correct the occlusal tilt; $2.5-\mathrm{mm}$ surgical extrusion in the right- molar area and 4-mm surgical impaction with maxillary advancement, BSSO and advancement genioplasty to advance the mandible and the chin.

A 3D model was not created for this patient because of her socio-economic status. Intermaxillary fixation was maintained for 4 weeks, and no relapse was observed during the 1-year- followup period. The treatment resulted in a more favorable symmetrical facial and lateral profile (Fig. 3B).

\section{Case 3}

The third patient was 18-years- old when he was admitted to our department. His medical history revealed that he had TMJ ankylosis on the left side due to a trauma experienced when he was 2 years old. Class II skeletal discrepancy and facial asymmetry had developed. His maximum mouth opening was reported to be $25 \mathrm{~mm}$ after the TMJ ankylosis surgery. He did not use any functional orthodontic appliance during the growth period. Clinically, facial asymmetry with a deviation to the left, a birdlike facial appearance, tilted occlusion, inequality of gingival show during smiling, a retrognathic mandibular appearance, a large overjet, and Angle class II dental malocclusion were observed (Fig. 4A). In the cephalometric analysis using a lateral cephalogram, mandibular retrognathia (SNB: $\left.68^{\circ}\right)$ with a-skeletal class II maxillomandibular relationship (ANB: $10^{\circ}$ ), and proclination of the upper and lower incisal teeth were determined (Table 1). In the posteroanterior cephalogram, the discrepancy between the left and the right maxillary height, and

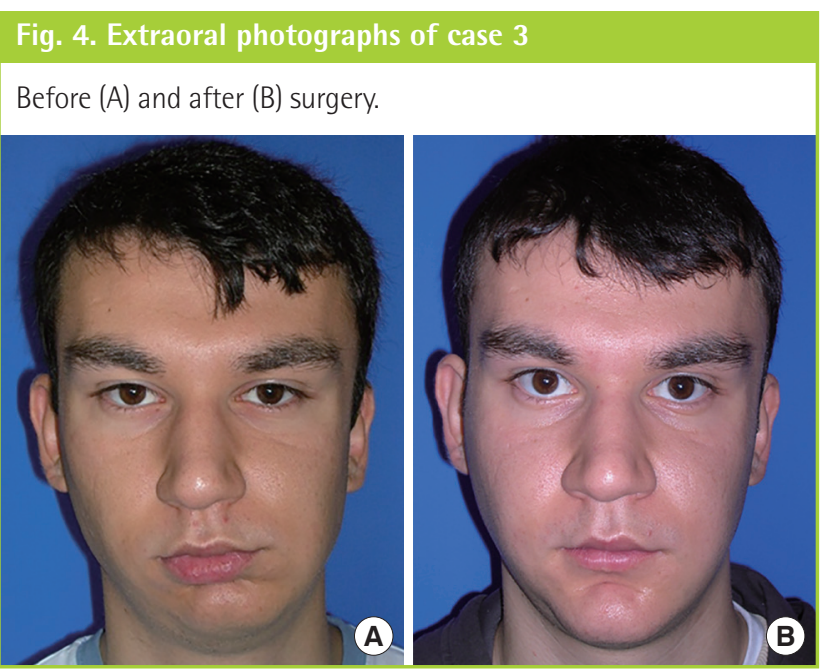

between the molar teeth and the orbital point was $11 \mathrm{~mm}$. The discrepancy between the left and the right mandibular ramal length was $15 \mathrm{~mm}$, and the deviation in the menton to the midline was $14 \mathrm{~mm}$ (Table 1). Deep antegonial notching was noted in the cephalograms.

\section{Surgical plan}

Le Fort 1 osteotomy to correct the occlusal tilt; 3-mm surgical impaction -in the right -molar area and 6-mm surgical impaction without maxillary advancement.

BSSO to advance and to correct the midline deviation of the mandible.

7-mm advancement -with genioplasty.

A 3D model was not created for this patient. Intermaxillary fixation was maintained for 4 weeks, and no relapse was observed during the 1-year-follow-up period. The treatment resulted in a more favorable symmetrical facial and lateral profile (Fig. 4B).

\section{Case 4}

A 22-year-old male patient was admitted to our department. He had a history of TMJ ankylosis with limitations in opening his mouth on the right side due to trauma he had experienced when he was 4 years old. The patient reported in his history that he had not used any functional appliance to maintain the mouth opening range and to correct his facial asymmetry. After diagnosis with a plain X-ray and CT, the patient underwent a surgery to release the ankylosis; the prefabricated condylar cap that was inserted previously in another center was excluded. His preoperative 10 -mm mouth opening became $25 \mathrm{~mm}$ after the surgery. A clinical examination revealed mandibular deviation to the right, maxillomandibular deviation to the right, occlusal tilting, and a bird-like facial appearance due to the retrognathic mandible. In 
Table 1. Cephalometric summary of cases

\begin{tabular}{|c|c|c|c|c|c|c|c|c|}
\hline \multirow{2}{*}{ Variable } & \multicolumn{2}{|c|}{ Case 1} & \multicolumn{2}{|c|}{ Case 2} & \multicolumn{2}{|c|}{ Case 3} & \multicolumn{2}{|c|}{ Case 4} \\
\hline & Pre-surgery & Post-surgery & Pre-surgery & Post-surgery & Pre-surgery & Post-surgery & Pre-surgery & Post-urgery \\
\hline \multicolumn{9}{|l|}{ Skeletal measurements } \\
\hline SNA $\left({ }^{\circ}\right)$ & 77 & 79 & 75 & 77.5 & 85 & 86 & 80.5 & 81 \\
\hline SNB $\left(^{\circ}\right)$ & 66.5 & 72 & 68 & 70 & 75 & 76 & 67.5 & 67.5 \\
\hline $\operatorname{ANB}\left({ }^{\circ}\right)$ & 11.5 & 7 & 7 & 7.5 & 10 & 10 & 13 & 13.5 \\
\hline SN/Go-Gn( $\left(^{\circ}\right)$ & 40 & 40 & 34.5 & 35 & 24 & 27 & 42 & 40 \\
\hline $\mathrm{N}-\mathrm{A} / \mathrm{FH}\left({ }^{\circ}\right)$ & 89 & 91.5 & 84 & 85 & 88 & 90 & 92 & 94 \\
\hline $\mathrm{N}-\mathrm{Pg} / \mathrm{FH}\left({ }^{\circ}\right)$ & 78 & 88 & 77 & 87 & 80 & 82 & 72 & 76.5 \\
\hline N-ANS (mm) & 53 & 53 & 51 & 50 & 57 & 57 & 59 & 58 \\
\hline ANS-Me (mm) & 75 & 75 & 69 & 70 & 67 & 74 & 63 & 63 \\
\hline $\mathrm{N}-\mathrm{Me}(\mathrm{mm})$ & 128 & 128 & 118 & 120 & 125 & 128 & 134 & 136 \\
\hline S-Go right (mm) & 78 & 72 & 78 & 78 & 97 & 94 & 82 & 79 \\
\hline S-Go left (mm) & 77 & 72 & 77 & 77 & 87 & 90 & 91 & 92 \\
\hline S-PNS (mm) & 46.5 & 45.5 & 52 & 46.5 & 53 & 55 & 49 & 50 \\
\hline PP-SN $\left({ }^{\circ}\right)$ & 12.5 & 10 & 4 & 7 & 8.5 & 4 & 11 & 16 \\
\hline Occlusal plane/SN $\left(^{\circ}\right)$ & 19 & 20 & 20 & 25 & 20 & 10 & 28 & 30 \\
\hline Co-Go right (mm) & 51 & 46 & 45.5 & 46 & 73 & 70 & 53 & 54 \\
\hline Co-Go left (mm) & 45.5 & & 51.5 & 51 & 54 & 55 & 63 & 60 \\
\hline Co-A right(mm) & 89 & 85 & 75.5 & 78 & 100 & 102 & 91 & 91 \\
\hline Co-A left (mm) & 83.5 & & 80 & 82 & 91 & 92 & 96 & 97 \\
\hline Co-Gn right (mm) & 96 & 109 & 88.5 & 101 & 120 & 128 & 101 & 110 \\
\hline Co-Gn left (mm) & 89.5 & & 96 & 109 & 105 & 115 & 108 & 108 \\
\hline Go-Me right (mm) & 54 & 73 & 53 & 66 & 75 & 82 & 59 & 65 \\
\hline Go-Me left (mm) & 51 & & 59 & 67.5 & 70 & 75 & 60 & 60 \\
\hline $\mathrm{N} \perp \mathrm{FH}-\mathrm{A}(\mathrm{mm})$ & -2 & -1 & -6.5 & -3 & 0 & 1 & 2 & 2 \\
\hline $\mathrm{N} \perp \mathrm{FH}-\mathrm{Pg}(\mathrm{mm})$ & -21 & -6.5 & -24.5 & -9.5 & -27 & -15 & -28 & -24 \\
\hline \multicolumn{9}{|c|}{ Dentoalveoler measurements } \\
\hline U1/NA $\left(^{\circ}\right)$ & 32 & 29 & 35 & 25 & 5 & 14 & 2 & 18 \\
\hline U1-NA (mm) & 8.5 & 9.5 & 11 & 6.5 & -2 & 1 & -2 & 0 \\
\hline $\mathrm{L} 1 / \mathrm{NB}\left({ }^{\circ}\right)$ & 44 & 41 & 40 & 47 & 30 & 30 & 50 & 40 \\
\hline L1-NB (mm) & 11 & 15.5 & 12 & 15 & 5 & 10 & 18 & 16 \\
\hline Overjet (mm) & 11 & 2.5 & 9 & 1.5 & 8 & 2 & 3 & 2 \\
\hline Overbite (mm) & 2.5 & 2 & 3 & 1.5 & 6 & 3.5 & 5 & 2 \\
\hline \multicolumn{9}{|c|}{ Soft tissue measurements } \\
\hline Nasolabial angle $\left({ }^{\circ}\right)$ & 106 & 120 & 91 & 98 & 100 & 95 & 128 & 128 \\
\hline Ls $\perp S n-P g$ & 4.5 & 0 & 7 & 2 & 7 & 4 & 6 & 4 \\
\hline $\mathrm{Li} \perp \mathrm{Sn}-\mathrm{Pg} g^{\prime}$ & 8 & 1 & 9 & 1 & 5 & -2 & 8,5 & 3 \\
\hline \multicolumn{9}{|c|}{ Posteroanterior measurements } \\
\hline Co-Go right (mm) & 59 & 54 & 39 & 47 & 69 & 68 & 60 & 64 \\
\hline
\end{tabular}

SNA, angle among sella $(\mathrm{S})$, nasion $(\mathrm{N})$, and A points (deepest point on the curved bony outline of anterior maxilla) that defines maxillary anteroposterior position to cranial base; SNB, angle among sella (S), nasion (N), and B points (deepest point on the curved bony outline of anterior mandible) defines that mandibular anteroposterior position to cranial base; ANB, angle among A, N, and B points that defines the position of maxilla in reference to mandible or vice versa; SN/Go-Gn, angle between sella-nasion and Gonion-Gnathion planes that defines mandibular plane angle to cranial base; N-A/FH, angle between nasion-A point and Frankfort horizontal plane that defines maxillary position; N-Pg/FH, angle between nasion-pogonion point and Frankfort horizontal plane that defines mandibular position; N-ANS, distance between nasion and anterior nasal spine that defines anterior maxillary height; ANS-Me, distance between anterior nasal spine and menton that defines lower anterior height; N-Me, distance between nasion and menton that defines anterior facial height; S-Go right, distance between sella and gonion that defines posterior facial height on the right side; S-Go left, distance between sella and gonion that defines posterior facial height on the left side; S-PNS, Distance between sella and posterior nasal spine that defines upper posterior maxillary height; PP/SN, angle between palatal plane and sella-nasion line (cranial base) that defines the palatal plane inclination; Occlusal plane/SN, angle between occlusal plane and sella-nasion line (cranial base) that defines the occlusal plane inclination; Co-Go right, distance between condylion and gonion that defines right ramus lenght; Co-Go left, distance between condylion and gonion that defines left ramus lenght; $\mathrm{Co}-\mathrm{A}$ right, distance between condylion and A point that defines relative maxillary lenght; Co-A left, distance between condylion and A point that defines relative maxillary lenght; Co-Gn right, distance between condylion and gnathion that defines relative mandibular lenght at right side; Co-Gn left, distance between condylion and gnathion that defines relative mandibular lenght at left side; Go-Me right, distance between gonion and menton that defines right corpus lenght; Go-Me left, distance between gonion and menton that defines left corpus lenght; $\mathrm{N} \perp \mathrm{FH}-\mathrm{A}$, distance from point $\mathrm{A}$ to nasion perpendecular (line from nasion to the chin perpendecular to Frankfort plane) line that defines maxillary position to cranial base; $\mathrm{N} \perp \mathrm{FH}-\mathrm{Pg}$, distance between pogonion to nasion perpendecular that defines mandibular position to cranial base; U1/NA, angle between upper incisor and nasion-A point line; U1-NA, distance between upper incisor and nasion-A point line; L1/NB, angle between lower incisor and nasion-B point line; L1-NB, distance between lower and nasion-B point line; Overjet, horizontal distance between upper and lower incisor teeth; Overbite, vertical distance between upper and lower incisor teeth; Nasolabial angle, angle between nasal tip-subnasal point line and subnasal point-soft tissue A point line; Ls $\perp S n-P g$ ', distance between point labrale superior and point subnasale and point pogonion; Li $\perp S n-P g$ ', distance between point labrale inferior and point subnasale and point pogonion; Co-Go right, distance between point condylion and point gonion. 
his intraoral examination, an increased overjet and class II malocclusion were noted. In the cephalometric evaluation, mandibular retrusion (SNB: 67.5 ) with a class II skeletal relationship (ANB: $13^{\circ}$ ) and high mandibular plane inclination (SN/GoGn: $42^{\circ}$ ), midline deviation, and asymmetric ramal heights were determined. The mandibular ramal height (Co-Go) was $101 \mathrm{~mm}$ on the right and $108 \mathrm{~mm}$ on the left. The maxillary heights between the left and the right orbital point and the maxillary first molars were $54 \mathrm{~mm}$ and $62 \mathrm{~mm}$, respectively (Table 1). Fixed orthodontic appliances were used for correcting the alignment problem and preparing for surgery. However, the patient did not want a long orthodontic treatment process; therefore, only leveling of the teeth with an orthodontic appliance was performed. A 3D model was created using the patient's CT images.

\section{Surgical plan}

Correction of maxillary tilting with impaction of the left segment $(7 \mathrm{~mm})$ at the level of the molar teeth to correct the asymmetric gingival show with Le Fort 1 surgery,

Correction of the midline deviation of the mandible with BSSO.

Advancement genioplasty.

Following the surgery, a fixed appliance was maintained and intermaxillary fixation was maintained for 4 weeks. During 1 -year of follow-up, no relapse was observed.

\section{DISCUSSION}

Facial asymmetry due to unilateral condylar ankylosis leads to aesthetic and functional problems. The general treatment plan for such anomalies, with the goal of achieving a symmetric facial appearance, is bimaxillary surgery with or without condylar surgery [5]. In these cases, severe facial asymmetry with a retrognathic chin appearance was determined to have occured due to unilateral TMJ ankylosis during the early childhood period. Earlier operations had been performed to release the ankylosis, to increase the limitation of the mouth opening, and to restore the TMJ function to activate the mandible as much as possible. Thus far, various treatment techniques, such as ramus osteotomy, high condylectomy, coronoidectomy, corticoid infusions, gap arthroplasty, bilateral arthrotomy, distraction osteogenesis, joint reconstruction with alloplastic prosthesis, free vascularized whole-joint transplants, early mobilization, and aggressive physiotherapy, have been reported for the treatment of TMJ ankylosis [6-8]. A hybrid appliance is an orthopedic treatment appliance that allows the growth of dentoalveolar structures in the affected hypoplastic side and is used for correcting mandibular deviation [9]. Mandibular advancement appliances are used for stimulating the forward growth of the mandible by bringing the lower jaw forward by at least $6 \mathrm{~mm}$ by adapting the condylar growth to the new position of the mandible during the growth period [10]. However, all these functional appliances are removable and the treatment success depends on the patient's cooperation. The treatment duration is 1 to 2 years. Even if the treatment is successful, the malformation can reoccur because of the ongoing asymmetrical growth potential following the functional treatment. After bimaxillary orthognathic surgery, the facial asymmetry disappeared and a relatively balanced lateral profile was obtained. The cephalometric results revealed that the- skeletal class II relationship decreased but was maintained (Table 1). In class II cases with a prominent retrognathic lower jaw, dentoalveolar compensation (lower teeth proclination) occured. This point must be considered while formulating the treatment plan, and decompensation should be performed by retracting the proclined teeth. An orthognathic surgical plan was not considered in very young patients because of the growth potential; thus, functional and fixed appliances could be used for achieving maximum interdigitation with class I occlusion. However, the treatment plan was not successful in cases 1 and 2 for the following 2 reasons: (1) the patients had not used the functional appliances properly, and (2) the treatment rate could not catch up with the growth rate. Orthognathic surgery was delayed until after the completion of growth. Orthognathic surgery is a very effective treatment choice if dentofacial orthopedic treatment does not overcome the problem at hand. Further, mandibular surgical movement could not be performed properly because of the proclination of the mandibular incisors, and advancement genioplasty was added to the surgical plan. In the $4 \mathrm{TMJ}$ ankylosis patients, antegonial notching was seen characteristically on the affected sides. This was probably the result of a pathological vertical reduction of the ramus height. Further, apposition in the gonial area due to abnormal muscle functions and bone remodeling should not be considered in TMJ ankylosis patients. The surgeon used 3D models of patients 1 and 4 to guide the surgical plan, and these models helped the surgeon to clearly observe the thickness of the mandible of the affected and the non-affected sides before surgery.

Good facial symmetry and a satisfactory facial profile were obtained with only orthognathic surgery in all 4 of these cases of facial asymmetry.

\section{CONFLICT OF INTEREST}

No potential conflict of interest relevant to this article was reported. 


\section{PATIENT CONSENT}

The patient provided written informed consent for the publication and the use of their images.

\section{REFERENCES}

1. Melnik AK. A cephalometric study of mandibular asymmetry in a longitudinally followed sample of growing children. Am J Orthod Dentofacial Orthop 1992;101:355-66.

2. Severt TR, Proffit WR. The prevalence of facial asymmetry in the dentofacial deformities population at the University of North Carolina. Int J Adult Orthodon Orthognath Surg 1997;12:171-6.

3. Bishara SE, Burkey PS, Kharouf JG. Dental and facial asymmetries: a review. Angle Orthod 1994;64:89-98.

4. Lindsay JS, Fulcher CL, Sazima HJ, et al. Surgical management of ankylosis of the temporomandibular joint: report of two cases. J Oral Surg 1966;24:264-70.

5. Sugawara Y, Hirabayashi S, Susami T, et al. The treatment of hemimandibular hyperplasia preserving enlarged condylar head. Cleft Palate Craniofac J 2002;39:646-54.

6. Epker BN, Stella JP, Fish LC. Dentofacial deformities: integrated orthodontic and surgical correction. St. Louis, MO: Mosby; 1996.

7. Mercuri LG. The use of alloplastic prostheses for temporomandibular joint reconstruction. J Oral Maxillofac Surg 2000;58:70-5.

8. Obwegeser HL, Hadjianghelou O. Two ways to correct bird-face deformity. Oral Surg Oral Med Oral Pathol 1987; 64:507-18.

9. Proffit WR, Fields HW, Sarver DM. Contemporary orthodontics. St. Louis, MO: Elsevier Mosby; 2006.

10. Ko EW, Huang CS, Chen YR, et al. Cephalometric craniofacial characteristics in patients with temporomandibular joint ankylosis. Chang Gung Med J 2005;28:456-66. 